\title{
RNA-seq Analysis of Meristem Cells Identifies the FaFT3 Gene as a Common Floral Inducer in Japanese Cultivated Strawberry
}

\author{
Khoem Koembuoy ${ }^{1}$, Shiori Hasegawa ${ }^{1}$, Shungo Otagaki ${ }^{1 * *}$, Hirokazu Takahashi ${ }^{1}$, \\ Soichiro Nagano ${ }^{2 * * *}$, Sachiko Isobe ${ }^{2}$, Katsuhiro Shiratake ${ }^{1}$ and Shogo Matsumoto ${ }^{1 *}$ \\ ${ }^{1}$ Graduate School of Bioagricultural Sciences, Nagoya University, Nagoya 464-8601, Japan \\ ${ }^{2}$ Kazusa DNA Research Institute, Kisarazu 292-0818, Japan
}

In Arabidopsis thaliana, the FLOWERING LOCUS T (FT) gene, acting as a floral promoter, is expressed and translated in leaves, and is then transported to the shoot apical meristem. In contrast, the expression pattern of the FaFT3 gene in the crown, which contains the shoot apical meristem, is coordinated with the initiation of the floral bud in the June-bearing type of cultivated strawberry (Fragaria $\times$ ananassa) 'Nyoho'. However, whether the FaFT3 protein functions as a floral promoter and whether the expression pattern of FaFT3 in the crown observed in 'Nyoho' is conserved in other strawberry cultivars are not known. In this study, we investigated the floral inducer activity of the FaFT3 gene isolated from the cultivated strawberry 'Tochiotome' using FaFT3-overexpressing transgenic Arabidopsis lines and performed expression analysis on the FaFT3 gene in the crown tip of 'Tochiotome'. Transgenic plants overexpressing the FaFT3 gene exhibited an earlyflowering phenotype under both long-day and short-day conditions. Conversely, induction of FaFT3 expression at the crown tip specifically under floral induction conditions was not observed. However, RNA-seq analysis of laser microdissected meristem cells before and after floral bud initiation clearly revealed that the FaFT3 gene is specifically expressed in floral meristem cells. These results suggest that the FaFT3 gene acts as a common floral promoter in June-bearing Japanese cultivated strawberries.

Key Words: floral induction, floral meristem, Fragaria, RNA-seq, shoot apical meristem.

\section{Introduction}

The cultivated strawberry (Fragaria $\times$ ananassa) is an octoploid plant $(2 \mathrm{n}=8 \mathrm{x}=56)$ speculated to originate from a cross between the octoploid wild species F. chiloensis and F. virginiana (Njuguna et al., 2013). Recently, the genome of Fragaria $\times$ ananassa 'Camarosa' was determined by combining short-read and long-read sequencing approaches. Phylogenetic analysis using 31 de novo assembled transcriptomes, which were comprised of 12 wild Fragaria species and four $F$. vesca subspecies, further revealed that four subgenomes of Fragaria $\times$ ananassa originated from

Received; August 22, 2019. Accepted; November 26, 2019.

First Published Online in J-STAGE on February 21, 2020.

Special Issue "Strawberry".

* Corresponding author (E-mail: shogo@agr.nagoya-u.ac.jp).

** Corresponding author (E-mail: sotagaki@agr.nagoya-u.ac.jp).

*** Present address: Forest Tree Breeding Center, Forestry and Forest Products Research Institute, Forest Research and Management Organization, 3809-1 Ishi, Juo-cho, Hitachi, Ibaraki 319-1301, Japan. the diploid Fragaria species F. nipponica, F. iinumae, $F$. viridis, and $F$. vesca subsp. bracteata. Interestingly, the $F$. vesca subgenome is dominant over the other three subgenomes, as the number of genes on the F. vesca subgenome is greater than that on other subgenomes and many dominantly expressed homoeologs have been mapped onto the F. vesca subgenome (Edger et al., 2019).

Most cultivated strawberries produced in Japan are June-bearing (or seasonal flowering) types. During the vegetative phase, strawberry plants form vegetative shoots known as runners from a compressed and thickened stem called a crown. When the vegetative to reproductive transition occurs, inflorescence is differentiated from the meristem cells located at the tip of the crown. Generally, in June-bearing strawberry plants, flowering is inhibited under high temperature conditions over $25^{\circ} \mathrm{C}$ and flowering is promoted under cool temperature conditions between $5^{\circ} \mathrm{C}$ and $15^{\circ} \mathrm{C}$, irrespective of the photoperiod (Heide et al., 2013; Ito and Saito, 1962). When the temperature is between $15^{\circ} \mathrm{C}$ and $25^{\circ} \mathrm{C}$, flowering is induced only under short- 
day conditions (Heide, 1977; Sønsteby and Heide, 2006). A cultivation technology called forcing cultivation, frequently used in Japan to expand the harvesting period, utilizes this flowering behavior in strawberry plants. In this approach, daughter plants at the vegetative phase are grown for about 20 days under short-day and night cooling conditions with a low nitrogen fertilizer in the summer to induce floral transition. However, observation of the floral meristem under a microscope is necessary before transplanting because the treatment has no effect if the treatment period is too short to induce floral initiation. In addition, prolonged treatment causes a negative effect on fruit production because flower numbers in transplanted daughter plants are significantly reduced (Morishita et al., 1992; Ueki et al., 1993). Since determining the floral differentiation stage under a microscope is a skilled and time-consuming job, a simple alternative method is required. Understanding the molecular mechanism of flowering induction in cultivated strawberry is the first step to achieve this goal.

FLOWERING LOCUS T (FT) is well known as a floral inducer. In the facultative long-day plant Arabidopsis, FT is expressed in the phloem companion cells of leaves under inductive long-day conditions (An et al., 2004). Translated FT protein is then transported to the shoot apical meristem (SAM) and binds to a bZIP transcription factor, FD. This FT-FD complex promotes the expression of the floral meristem identity genes like APETALA1 (APl) (Abe et al., 2005). In contrast, TERMINAL FLOWER1 (TFL1) expressed in the SAM acts as a floral repressor by forming a complex with FD, preventing binding of FT to FD (Abe et al., 2005). However, in strawberry, the situation is complicated. Although Rantanen et al. (2014) reported that the ectopic expression of $F v F T 1$ in the long-day accession of $F$. vesca promoted flowering, expression of the FvFT1 gene in leaves was observed only under non-floweringinductive long-day conditions in the short-day accession of F. vesca (Koskela et al., 2012). In the Junebearing cultivated strawberry 'Nyoho', three FT homologues were identified (FaFT1-3), but none of them exhibited an expression pattern corresponding to the vegetative-to-reproductive transition in leaves. Instead, FaFT3 exhibited an expression pattern correlated with floral induction in the shoot tip containing SAM (Nakano et al., 2015). However, whether the expression pattern of FaFT3 is conserved among other Junebearing strawberry cultivars and whether FaFT3 functions as a floral inducer are not known.

Previously, we isolated FaFT1 cDNA from the Junebearing cultivated strawberry 'Tochiotome' and confirmed that FaFT1 was expressed in leaves under nonflowering-inductive long-day conditions (Nakajima et al., 2014). However, we failed to isolate the FaFT2 and FaFT3 genes at that time. In the present study, we cloned FaFT3 from 'Tochiotome' with the aid of the
FaFT3 sequence of 'Nyoho' and the database of the octoploid strawberry genome (Strawberry GARDEN; http:// strawberry-garden.kazusa.or.jp/index.html; Hirakawa et al., 2014) and compared its expression pattern between meristem cells at the vegetative or reproductive phases. In addition, floral inducer activity of FaFT3 was investigated using transgenic Arabidopsis plants ectopically expressing FaFT3. Our results support the idea that FaFT3 acts as a common floral inducer in Junebearing Japanese cultivated strawberries.

\section{Materials and Methods}

\section{Plant materials}

Parent plants of Fragaria $\times$ ananassa 'Tochiotome' and 'Akihime' were grown in a greenhouse equipped with a misting system under natural day-length conditions at Aichi Agricultural Research Center in 2015. The misting system was activated when the temperature in the greenhouse reached $30^{\circ} \mathrm{C}$ or higher. 'Tochiotome' and 'Akihime' runner plants with three expanded leaves were cut off and planted in pots on 30 May 2015. We removed the leaves from the plants except for three to five sets of the youngest fully developed leaves. Plants were grown under short-day and low temperature conditions (SDLT; an 8-h photoperiod and day/night temperatures of average $32.5 / 18.7^{\circ} \mathrm{C}$ ) or long-day and high temperature conditions (LDHT; average day length and day/night temperatures were 13 hours 49 minutes and $31.2 / 27.4^{\circ} \mathrm{C}$, respectively). SDLT plants were grown in a field in the daytime, but were transferred into a curtained tunnel tent with air cooling from 17:00 to 9:00. We sampled leaves and crowns of 'Tochiotome' plants on $0,5,15,20,25,30,35$, and the $41^{\text {st }}$ day after SDLT or LDHT treatments. The youngest expanded leaf was sampled from each plant at 17:00. Tissues were stored at $-80^{\circ} \mathrm{C}$. The developmental stages of the flower buds were defined as: stage $0-$ an undifferentiated stage, stage $\mathrm{A}-$ an initial to late differentiation stage.

\section{Total RNA extraction from a $5 \mathrm{~mm}$ crown tip}

Total RNA was extracted from a tip of the strawberry crown ( $5 \mathrm{~mm}$ in length) by the hot borate method, as described in Nakajima et al. (2014).

\section{Isolation of FaFT3 and FaFD}

The FaFT3 and FaFD genes of 'Tochiotome' were isolated using a standard RT-PCR method described by Nakajima et al. (2014). Briefly, total RNA extracted from the tip of the crown was reverse-transcribed using a PrimeScript RT reagent Kit with gDNA Eraser (Perfect Real Time) (TaKaRa Bio Inc., Shiga, Japan). Subsequently, $1 \mu \mathrm{L}$ of complementary DNA (cDNA) was used for PCR with Ex-Taq (TaKaRa Bio). The FaFD gene of 'Akihime' was isolated by the same procedure. Primers used for the analysis are listed in Supplemental Table S1. 
Vector construction and plant transformation

Full-length coding sequences of FaFT1 or FaFT3 were amplified and cloned into a T-Vector pMD20 (TaKaRa Bio). Then, plasmids were digested with NdeI and $K p n I$ and ligated into the binary vector pRI 101-AN (TaKaRa Bio). The resulting vectors, pRI 101-ANFaFT1 and pRI 101-AN-FaFT3, were transferred into Agrobacterium tumefaciens strain GV3101 by electroporation. Arabidopsis thaliana ecotype Columbia were transformed using the modified floral dip method (Narusaka et al., 2010). Transgenic plants were grown under a long-day (16-h photoperiod) or a short-day (8-h photoperiod) at $22^{\circ} \mathrm{C}$. We analyzed the flowering phenotype in the second generation.

Total RNA extraction from laser microdissection (LM)isolated tissues

Laser microdissection was performed according to Takahashi et al. (2015) with the following modifications: tips of the strawberry crown (3 $\mathrm{mm}$ in length) were sectioned at a thickness of $12 \mu \mathrm{m}$ and SAM or the floral meristem were collected from crown crosssections using a Veritas Laser Microdissection System LCC1704 (Molecular Devices, Sunnyvale, CA, USA). Total RNA was extracted from the LM-isolated tissues using a PicoPure RNA isolation kit (Thermo Fisher Scientific, Waltham, MA, USA) according to the manufacturer's protocol. The quantity and value of the RNA Integrity Number (RIN) of extracted total RNA was assessed using the Quant-iT RiboGreen RNA Reagent (Invitrogen, Carlsbad, CA, USA) and an RNA 6000 Pico Kit on an Agilent 2100 Bioanalyzer (Agilent Technologies, Santa Clara, CA, USA). RNA samples with a RIN value more than 5.6 were used for RNA-seq analysis.

\section{RNA-seq library preparation}

cDNA was generated using a SMART-Seq v4 Ultra Low Input RNA Kit for Sequencing (TaKaRa Bio USA, Inc., Mountain View, CA, USA) from $3 \mathrm{ng}$ each of RNA samples. Ten cycles of PCR amplification were performed, the quantity of cDNA was analyzed with a Qubit dsDNA HS Assay Kit (Invitrogen), and then the cDNA was fragmented for $10 \mathrm{~min}$ by a Covaris sonicator (Covaris, Woburn, MA, USA). Using $5 \mathrm{ng}$ each of cDNA, sequencing libraries were prepared using the Low Input Library Prep Kit v2 (TaKaRa Bio USA) according to the manufacturer's protocol. We used nuclease-free water instead of total RNA as a negative control in cDNA amplification and library preparation, and confirmed that the concentration of the sequencing library of a negative control was below the detection limit. Paired-end $100 \mathrm{bp}$ sequencing was used to sequence the libraries on a HiSeq 2000 sequencer (Illumina, San Diego, CA, USA). All generated sequence data are available from the DNA Data Bank of Japan (DRX178207-DRX178213).

\section{RNA-seq data analysis}

Low quality RNA-seq reads were removed by a FASTX-Toolkit (http://hannonlab.cshl.edu/fastx_toolkit/ index.html) with the following options; -t 20 and -1 36. Reads mapped with Bowtie2 (Langmead and Salzberg, 2012) to the rRNA genes of $A$. thaliana were excluded. Quality trimmed and rRNA-contamination-filtered reads were aligned to the FANhybrid_r1.2 genome using Bowtie2. Clustering analysis of sequenced samples was performed using the TCC package in R (Tang et al., 2015). Differentially expressed genes (DEGs) between the SAM (S25-0-1, S25-0-2, and L25-0) and the floral meristem (S41-A-1 and S41-A-2) were identified using DESeq2 (Love et al., 2014). Genes with a FDR < 0.05 were classified as DEGs. For GO enrichment analysis, Arabidopsis homologues of each DEG were obtained from the Strawberry GARDEN and were added to the query list of agriGO v2.0 (Tian et al., 2017). Annotation data from TAIR10 were used as a reference.

\section{Quantitative real-time PCR ( $q P C R$ )}

Total RNAs extracted from the tip of the crown or LM-isolated tissues were reverse-transcribed using a PrimeScript RT reagent Kit with a gDNA Eraser (Perfect Real Time) (TaKaRa Bio). qPCR was carried out as described in Nakajima et al. (2014). Primers used for the analysis are listed in Supplemental Table S1.

\section{Results}

Functional analyses of the FaFT3 gene isolated from the cultivar 'Tochiotome'

To analyze whether the FaFT3 gene acts as a floral inducer in June-bearing type cultivated strawberries, we cloned it from the reproductive stage (30 days after SDLT treatment) of 'Tochiotome' crown tissue using primers corresponding to FaFT3 of 'Nyoho'. The isolated gene FaFT3_Tochi showed five nucleotide differences with two amino acid substitutions compared to the amino acid sequence of FaFT3 in 'Nyoho' (Figs. 1a and S1a). We generated transgenic Arabidopsis plants overexpressing FaFT3_Tochi, FaFT1 from 'Tochiotome' or a floral activator of wild strawberry FvFT1 from the $F$. vesca subsp. vesca accession Hawaii-4 under the control of a CaMV 35S promoter and investigated their flowering times under long-day and short-day conditions. As a result, 35S::FaFT3 Tochi flowered significantly earlier than wild type plants under both long-day and short-day conditions, whereas 35S::FvFT1 plants exhibited an early flowering phenotype only under short-day conditions (Fig. 1b, c). Surprisingly, although there is only one amino acid difference at position 80 between FvFT1 and FaFT1, flowering time was significantly delayed in 35S::FaFT1 plants under short-day conditions (Fig. 1a, c).

Expression pattern of FaFT3_Tochi in crown tissue The early flowering phenotype of 35S::FaFT3_Tochi 
(a)

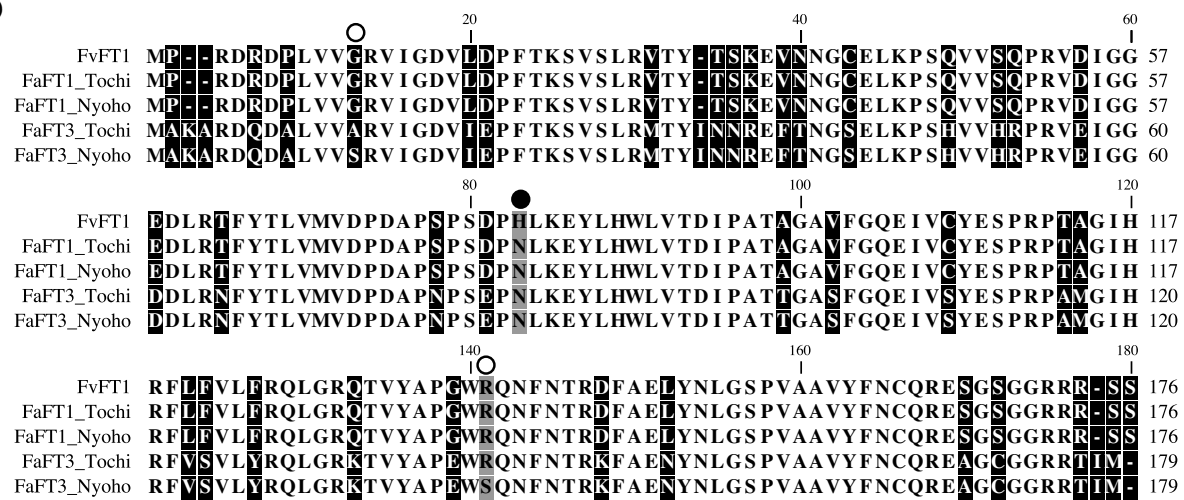

(b)

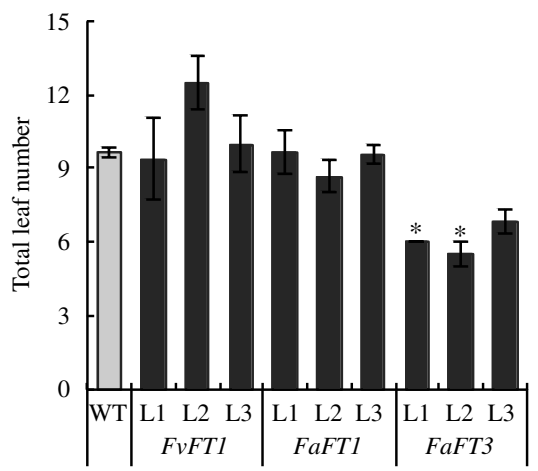

(c)

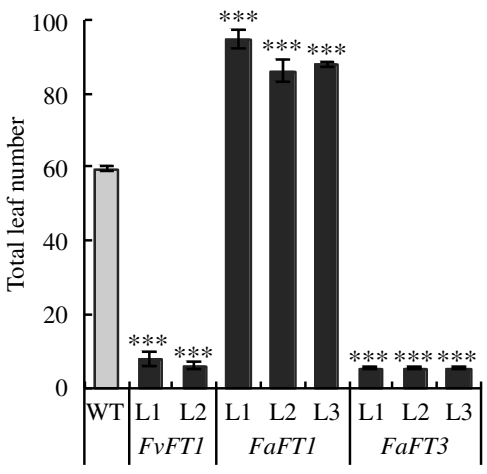

Fig. 1. Flowering phenotype of transgenic Arabidopsis plants ectopically expressing FT homologues from wild and cultivated strawberries. (a) Alignment of FT proteins isolated from a wild strawberry (AEP23098.1 for FvFT1) and the cultivated strawberries 'Tochiotome' and 'Nyoho' (BAR63708.1 for FaFT1_Nyoho and BAR63710.1 for FaFT3_Nyoho). Black or white circles represent amino acid polymorphisms between FvFT1 and FaFT1s or between FaFT3_Tochi and FaFT3_Nyoho, respectively. (b) and (c) Rosette leaf number at flowering of wild type (WT) and transgenic plants ectopically expressing FvFT1, FaFT1 or FaFT3 under long-day (b) and short-day (c) conditions. The vertical axis indicates the number of rosette leaves at flowering and the bars represent average values $\pm \mathrm{SE}(\mathrm{n}=3-6)$. Asterisks indicate significant differences between WT and transgenic plants (Dunnett's test, ${ }^{*} P<0.05, * * * P<0.001$ ). L1, L2, L3: the transgenic line 1-line 3.

transgenic plants suggests that FaFT3_Tochi acts as a floral inducer in June-bearing cultivated strawberry. To verify this hypothesis, we investigated the expression pattern of FaFT3_Tochi in the crown tissue under floral inductive conditions (SDLT) or vegetative conditions (LDHT). The expression pattern of the floral indicator gene FaAPl confirmed that the transition from the vegetative to reproductive phase occurred only under the SDLT conditions (Fig. 2). The expression pattern of FaFT3_Tochi was highly correlated with that of FaAP1. Both genes were down-regulated during the early to mid-phase (from day 0 to day 15 for FaFT3_Tochi and from day 0 to day 25 for FaAP1), but up-regulated during the mid- to late phase (from day 20 for FaFT3_Tochi and from day 30 for FaAP1) under the SDLT condition. However, expression of FaFT3_Tochi was also up-regulated transiently during the early to mid-phase, but was relatively low during the late phase under the LDHT condition (Fig. 2).
Isolation of FaFD and its expression patterns in crown tissue of the June-bearing cultivated strawberry 'Tochiotome'

As the expression pattern of FaFT3 could not fully explain the transition from the SAM to the floral meristem, we isolated $F a F D$, a putative binding partner of FaFT3, and investigated its expression pattern in the crown tissue of 'Tochiotome'. Two FD homologues were predicted in the strawberry GARDEN (FANhyb icon00001611_a.1.g00001.1 and FANhyb_rscf00000064. 1.g00025.1), and we succeeded in isolating two types of cDNA clones, classified as FaFD_allele_a and $F a F D$ allele_b. Both these sequences were very similar to FANhyb_icon00001611_a.1.g00001.1, and contained four nucleotide differences that caused two nonsynonymous substitutions at the coding region (Fig. S1b, c). Although the total homology of their deduced amino acid sequences and FD proteins from other plants was low, high homology was observed at the basic-leucine zipper domain (Fig. S1c). However, the 14-3-3 protein-binding region that is highly con- 

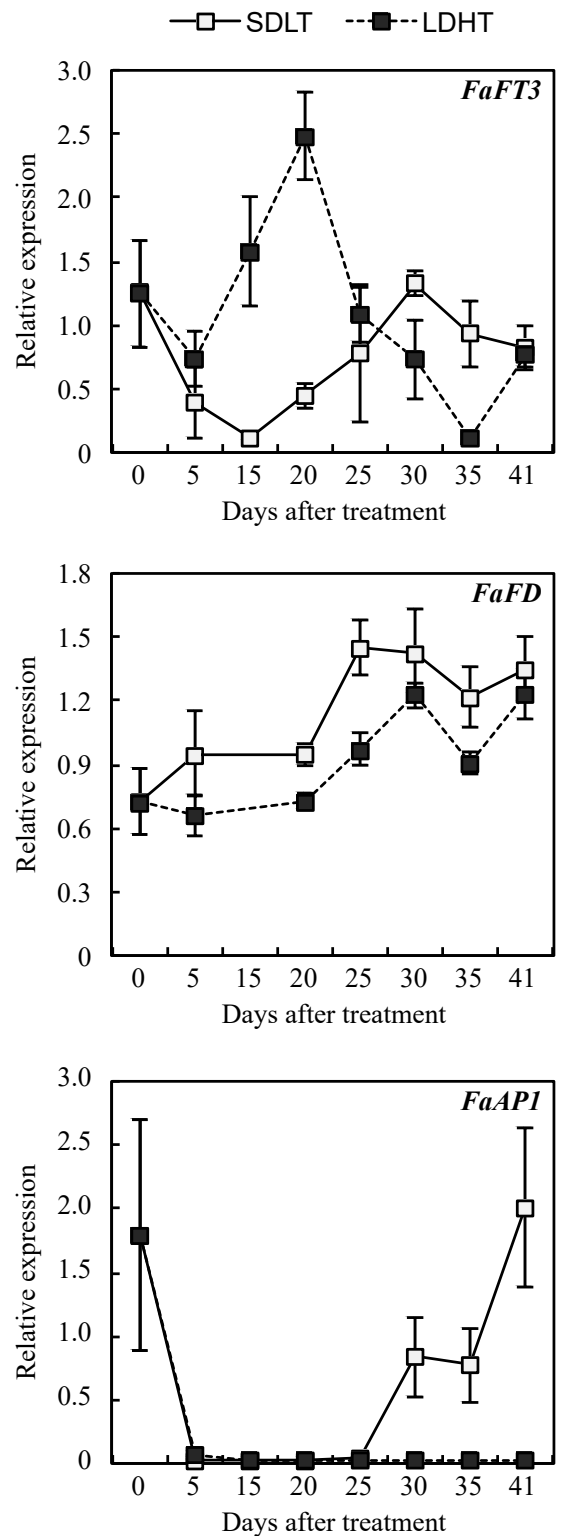

Fig. 2. Time-course expressions of the genes FaFT3, FaFD, and $F a A P 1$ in the crown tip of 'Tochiotome' grown under different photoperiods and temperatures for 0 to 41 days. The MSI gene was used as an internal control. The means $\pm \mathrm{SE}$ from three biological replicates are shown. SDLT, short-day, and low temperature condition; LDTH, long day and high temperature condition.

served in FD homologues (Taoka et al., 2011) was disrupted in the FaFD alleles (Fig. S1c). The disruption of the 14-3-3 protein-binding region was also observed in FaFD isolated from the cultivated strawberry 'Akihime' (Fig. S1c). From qPCR analysis, FaFD was expressed uniformly in the crown tissue under both SDLT and LDHT conditions (Fig. 2).

RNA-seq analysis of meristem cells during the transition from the vegetative to reproductive phase in a June-bearing strawberry

From the time-course expression analysis of FaFT3, it was speculated that $F a F T 3$ was a floral inducer of the June-bearing type of cultivated strawberry. However, FaFT3 expression in the crown tissue was observed not only during flower inductive conditions (SDLT), but also during flower non-inductive conditions (LDHT) (Fig. 2). To confirm the function of FaFT3 in meristem cells of the crown tissue and to identify other genes related to the vegetative-to-reproductive transition in cultivated strawberry, total RNA was extracted from lasermicrodissected meristem cells (Fig. S2a) and used for sequencing. We generated six sequence libraries that were then divided into four vegetative phase samples and two reproductive phase samples. For vegetative phase samples, we used a single plant grown under LDHT conditions for 25 or 41 days (LDHT25-0 and LDHT41-0) and two plants grown under SDLT conditions for 25 days (SDLT25-0-1 and SDLT25-0-2). Two plants grown under SDLT conditions for 41 days (SDLT41-A-1 and SDLT-A-2) were used as the reproductive phase samples. Microscopy observations confirmed that the meristems of all vegetative phase samples had a flat surface, whereas those of all reproductive phase samples had a dome-like structure. However, the number of total reads obtained from LDHT41-0 was about four to five times lower than the other five samples and LDHT41-0 formed a completely different clade by clustering analysis of the RNA-seq samples (Fig. S2b and data not shown). Therefore, we excluded LDHT41-0 from the subsequent analysis.

Using the DESeq2 package, we identified 1,151 DEGs between the vegetative and reproductive phase (Fig. S2c). Among them, 702 genes were up-regulated during the vegetative-to-reproductive transition. Although Kurokura et al. (2006) reported that Histone H4 mRNA was highly expressed in the central zone of the shoot apex in plants grown under floral inductive conditions, but not in plants grown under non-floral inductive conditions, we did not detect any significant differences in the expression of three Histone $\mathrm{H} 4$ genes between the vegetative and reproductive meristem (Table 1). GO enrichment analysis revealed that the biological processes "response to abiotic stimulus", "response to stimulus", and "response to endogenous stimulus" were the most strongly enriched. Up-regulated genes were also enriched in the biological processes "flower development" and "reproductive structure development" (Fig. 3a and data not shown). Notably, the FaFT3 gene (FANhyb icon00009135 a.1.g00001.1; Fig. S3) and the FaAPl gene (FANhyb_rscf00000019.1.g00010.1; Fig. S3) were classified as up-regulated genes (Table 1). In contrast, 449 down-regulated genes were strongly enriched in the biological processes "nucleobasecontaining compound metabolic process" and "nitrogen compound metabolic process". The GO term "reproductive structure development" was also enriched in down-regulated genes (Fig. 3b). The expression pattern of the three genes, FaFT3, FaFD, and FaAP1, in meri- 


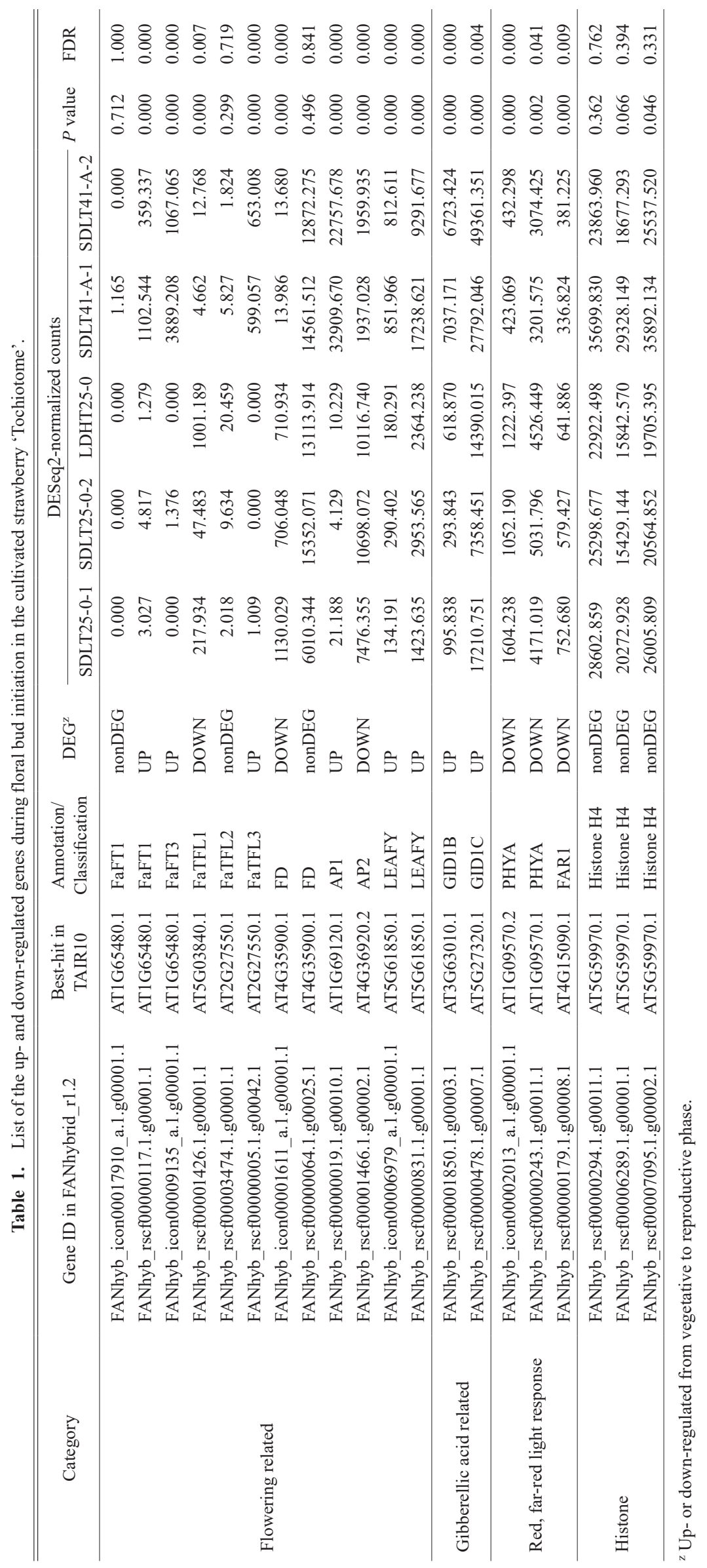


(a)

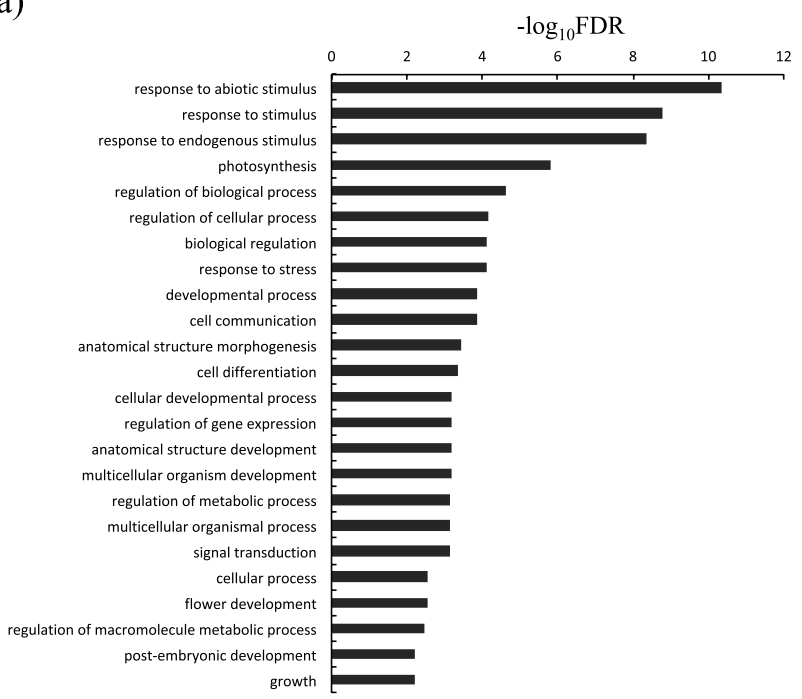

(b)

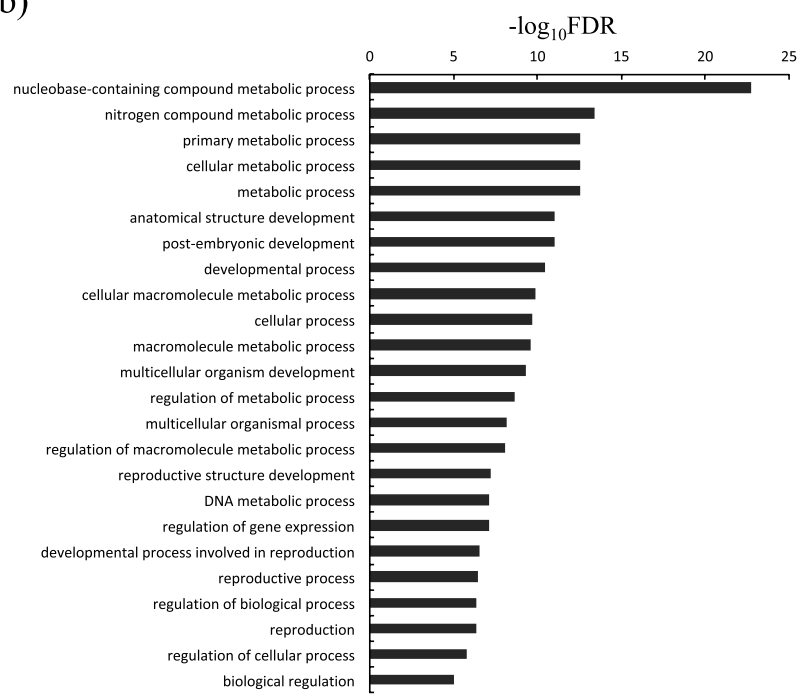

Fig. 3. Gene ontology enrichment analysis of the differentially expressed genes during floral bud initiation in the cultivated strawberry 'Tochiotome'. (a) Up-regulated from the vegetative to reproductive phase in meristem cells. (b) Down-regulated from the vegetative to reproductive phase in meristem cells.

stem cells was investigated by qPCR, and the results matched those of the RNA-seq data (Table 1; Fig. S4).

\section{Discussion}

In the present study, we isolated the FaFT3 gene from the cultivated strawberry 'Tochiotome' and examined whether it plays a major role in the transition of meristem cells from the vegetative to reproductive phase. Previously, Nakano et al. (2015) reported that FaFT3 gene expression was significantly up-regulated at the shoot tip when the plants were grown under short-day or low temperature conditions, and this upregulation of FaFT3 occurred prior to the induction of FaAP1 gene expression in the cultivated strawberry 'Nyoho'. In 'Tochiotome', no clear up-regulation of the FaFT3 gene specific to the SDLT treatment was observed (Fig. 2). This was in contrast to the induction of FaAP1 expression that was observed only in plants grown under a flowering-inductive SDLT treatment such as 'Nyoho'. However, in the RNA-seq analysis of meristem cells, the FaFT3 gene (FANhyb icon00009135 a.1.g00001.1) was significantly up-regulated during the transition from the vegetative to reproductive phase. This result suggests that the tissue where induction of the FaFT3 gene occurred was more spatially restricted to the meristem cells in 'Tochiotome' compared to 'Nyoho'. In addition to the similarity of the FaFT3 expression pattern between 'Tochiotome' and 'Nyoho', ectopic expression of FaFT3_Tochi in Arabidopsis promoted flowering, and the amino acid sequences of FaFT3 from the two cultivars were almost identical (Fig. 1). These results suggest that FaFT3 acts as a common floral inducer in June-bearing Japanese cultivated strawberries.
Involvement of other flowering-related genes in initiation of the floral bud in cultivated strawberry

During the vegetative to reproductive transition, FANhybrid r1.2 annotated genes encoding FaFT1, FaTFL3, AP1, and LEAFY were up-regulated similar to the FaFT3 gene, whereas annotated genes encoding FaTFL1, one of two FDs (FANhyb_icon00001611_a.1. g00001.1) and AP2 were down-regulated (Table 1). The expression patterns of $L E A F Y$ homologues and the $A P 2$ homologue agree with the previously described function of these two genes in the flowering of Arabidopsis. LEAFY plays a pivotal role in floral induction, whereas AP2 directly induces the transcription of the floral repressor AGAMOUS-LIKE 15 and represses the expression of floral activators such as SUPPRESSOR OF OVEREXPRESSION OF CONSTANS1 (Blázquez et al., 1997; Weigel et al., 1992; Yant et al., 2010). Similarly, it was recently shown that FD transcription was reduced soon after the floral transition in Arabidopsis (Abe et al., 2019). However, as the other FD homologue (FANhyb_rscf00000064.1.g00025.1) was stably expressed during the floral transition (Table 1), additional experiments are needed to clarify whether the FT-FD complex functions as a transient stimulus for floral initiation in cultivated strawberries.

We previously reported that a reduction in FaTFL2 expression in the tip of the crown is a key signal for floral initiation of 'Tochiotome' (Nakajima et al., 2014). However, our RNA-seq data revealed that an annotated gene encoding FaTFL1, not FaTFL2, was significantly down-regulated in the reproductive meristem compared to the vegetative meristem (Table 1). This result is consistent with the previous study of Nakano et al. (2015) showing that the expression of the FaTFL1 gene at the shoot tip of 'Nyoho' was significantly higher under 
non-floral inductive conditions than under floral inductive conditions. To determine whether either or both FaTFL1 and FaTFL2 act as floral repressors, flowering behavior should be observed in cultivated strawberry plants overexpressing or suppressing the FaTFL1 or FaTFL2 gene.

Other gene expression changes in meristem cells during the vegetative to reproductive transition in the cultivated strawberry 'Tochiotome'

In Arabidopsis, five major pathways (i.e., age pathway, autonomous pathway, gibberellin (GA) pathway, photoperiod pathway, and vernalization pathway) are involved in flowering time regulation (Teotia and Tang, 2015). In accordance with this, we detected an upregulation of the gibberellin receptors GIBBERELLININSENSITIVE DWARF1B (GID1B) and GIDIC homologues and a down-regulation of phytochrome $\mathrm{A}$ (phyA)-related genes (Table 1).

GID1 is a GA receptor involved in growth regulation through the formation of the GA-GID1-DELLA complex (Wang and Deng, 2014). In Arabidopsis, flowering is accelerated by exogenous GA treatment. However, the effect of GA treatment on strawberry plants is enigmatic because as Sonam and Singh (2018) reported, GA treatment could promote vegetative growth (i.e., runner production) in some experiments and could induce flowering in others. Hormonome analysis of the crown at the stage before and after floral bud induction could be useful to identify the phytohormones involved in the floral transition in cultivated strawberry.

PhyA is a phytochrome that primarily responds to red and far-red light, and FAR-RED-IMPAIRED RESPONSE1 (FAR1) is a transcription factor positively regulating the phyA-signaling pathway. PhyA exists in two photo-interconvertible forms termed $\mathrm{Pr}$ and $\mathrm{Pfr}$, which absorb red light and far-red light, respectively (Sheerin and Hiltbrunner, 2017; Wang and Wang, 2015). Interestingly, illumination of the crowns of the cultivated strawberry 'Strawberry Festival' with red light resulted in a significant decrease in the number of flowering plants (Takeda et al., 2008). Taking into account the fact that the strawberry cultivar 'Carmine' maintained at a high plant density during the vegetative phase flowered under flower-noninductive high temperature and long-day conditions (Takeda and Newell, 2006), Takeda et al. (2008) proposed that a high Pr:Pfr ratio would favor the activation of the flowering pathway in cultivated strawberry. Our finding that phyArelated genes are highly expressed in the vegetative meristem of 'Tochiotome' suggests that cultivated strawberries perceive the photoperiodic stimuli not only in leaves, but also in the crown harboring the vegetative meristem.

\section{Acknowledgements}

We are grateful to Mr. Yasushi Kondo for the plant material and Dr. Takeshi Kurokura for Arabidopsis plants overexpressing the $F v F T 1$ gene. This research was supported by JSPS KAKENHI Grant Numbers JP16K07594 and JP19K06031, and the Adaptable and Seamless Technology Transfer Program (A-STEP) (AS262Z02410N) from the Japan Science and Technology Agency (JST).

\section{Literature Cited}

Abe, M., Y. Kobayashi, S. Yamamoto, Y. Daimon, A. Yamaguchi, Y. Ikeda, H. Ichinoki, M. Notaguchi, K. Goto and T. Araki. 2005. FD, a bZIP protein mediating signals from the floral pathway integrator FT at the shoot apex. Science 309: 10521056.

Abe, M., S. Kosaka, M. Shibuta, K. Nagata, T. Uemura, A. Nakano and H. Kaya. 2019. Transient activity of the florigen complex during the floral transition in Arabidopsis thaliana. Development 146: dev171504. DOI: 10.1242/dev.171504.

An, H., C. Roussot, P. Suárez-López, L. Corbesier, C. Vincent, M. Piñeiro, S. Hepworth, A. Mouradov, S. Justin, C. Turnbull and G. Coupland. 2004. CONSTANS acts in the phloem to regulate a systemic signal that induces photoperiodic flowering of Arabidopsis. Development 131: 36153626.

Blázquez, M. A., L. N. Soowal, I. Lee and D. Weigel. 1997. LEAFY expression and flower initiation in Arabidopsis. Development 124: 3835-3844.

Edger, P. P., T. J. Poorten, R. VanBuren, M. A. Hardigan, M. Colle, M. R. McKain, R. D. Smith, S. J. Teresi, A. D. L. Nelson, C. M. Wai, E. I. Alger, K. A. Bird, A. E. Yocca, N. Pumplin, S. Ou, G. Ben-Zvi, A. Brodt, K. Baruch, T. Swale, L. Shiue, C. B. Acharya, G. S. Cole, J. P. Mower, K. L. Childs, N. Jiang, E. Lyons, M. Freeling, J. R. Puzey and S. J. Knapp. 2019. Origin and evolution of the octoploid strawberry genome. Nat. Genet. 51: 541-547.

Heide, O. M. 1977. Photoperiod and temperature interactions in growth and flowering of strawberry. Physiol. Plant. 40: 2126.

Heide, O. M., J. A. Stavang and A. Sønsteby. 2013. Physiology and genetics of flowering in cultivated and wild strawberries - a review. J. Hortic. Sci. Biotechnol. 88: 1-18.

Hirakawa, H., K. Shirasawa, S. Kosugi, K. Tashiro, S. Nakayama, M. Yamada, M. Kohara, A. Watanabe, Y. Kishida, T. Fujishiro, H. Tsuruoka, C. Minami, S. Sasamoto, M. Kato, K. Nanri, A. Komaki, T. Yanagi, Q. Guoxin, F. Maeda, M. Ishikawa, S. Kuhara, S. Sato, S. Tabata and S. N. Isobe. 2014. Dissection of the octoploid strawberry genome by deep sequencing of the genomes of Fragaria species. DNA Res. 21: 169-181.

Ito, H. and T. Saito. 1962. Studies on the flower formation in the strawberry plants I. Effects of temperature and photoperiod on the flower formation. Tohoku J. Agric. Res. 13: 191-203.

Koskela, E. A., K. Mouhu, M. C. Albani, T. Kurokura, M. Rantanen, D. J. Sargent, N. H. Battey, G. Coupland, O. Elomaa and T. Hytönen. 2012. Mutation in TERMINAL FLOWER1 reverses the photoperiodic requirement for flowering in the wild strawberry Fragaria vesca. Plant Physiol. 159: 1043-1054.

Kurokura, T., Y. Inaba and N. Sugiyama. 2006. Histone H4 gene expression and morphological changes on shoot apices of strawberry (Fragaria $\times$ ananassa Duch.) during floral induction. Sci. Hortic. 110: 192-197.

Langmead, B. and S. L. Salzberg. 2012. Fast gapped-read align- 
ment with Bowtie 2. Nat. Methods 9: 357-359.

Love, M. I., W. Huber and S. Anders. 2014. Moderated estimation of fold change and dispersion for RNA-seq data with DESeq2. Genome Biol. 15: 550. DOI: 10.1186/s13059-0140550-8.

Morishita, M., K. Ide, T. Mochizuki and U. Noguchi. 1992. Induction of axillary flower bud by low-night-temperature with short-day treatment in strawberry. Kyushu Agr. Res. 54: 201 (In Japanese).

Nakajima, R., S. Otagaki, K. Yamada, K. Shiratake and S. Matsumoto. 2014. Molecular cloning and expression analyses of FaFT, FaTFL, and FaAP1 genes in cultivated strawberry: their correlation to flower bud formation. Biol. Plant. 58: 641-648.

Nakano, Y., Y. Higuchi, Y. Yoshida and T. Hisamatsu. 2015. Environmental responses of the $F T / T F L 1$ gene family and their involvement in flower induction in Fragaria $\times$ ananassa. J. Plant Physiol. 177: 60-66.

Narusaka, M., T. Shiraishi, M. Iwabuchi and Y. Narusaka. 2010. The floral inoculating protocol: a simplified Arabidopsis thaliana transformation method modified from floral dipping. Plant Biotechnol. 27: 349-351.

Njuguna, W., A. Liston, R. Cronn, T. L. Ashman and N. Bassil. 2013. Insights into phylogeny, sex function and age of Fragaria based on whole chloroplast genome sequencing. Mol. Phylogenet. Evol. 66: 17-29.

Rantanen, M., T. Kurokura, K. Mouhu, P. Pinho, E. Tetri, L. Halonen, P. Palonen, P. Elomaa and T. Hytönen. 2014. Light quality regulates flowering in FvFT1/FvTFL1 dependent manner in the woodland strawberry Fragaria vesca. Front. Plant Sci. 5: 271.

Sheerin, D. J. and A. Hiltbrunner. 2017. Molecular mechanisms and ecological function of far-red light signalling. Plant Cell Environ. 40: 2509-2529.

Sonam and S. K. Singh. 2018. A review on impact of $\mathrm{GA}_{3}$ application on strawberry cultivation. Int. J. Chem. Stud. 6: $1382-1387$.

Sønsteby, A. and O. M. Heide. 2006. Dormancy relations and flowering of the strawberry cultivars Korona and Elsanta as influenced by photoperiod and temperature. Sci. Hortic. 110: $57-67$.

Takahashi, H., T. Yamauchi, I. Rajhi, N. K. Nishizawa and M. Nakazono. 2015. Transcript profiles in cortical cells of maize primary root during ethylene-induced lysigenous aerenchyma formation under aerobic conditions. Ann. Bot. 115: 879-894.

Takeda, F. and M. Newell. 2006. A method for increasing fall flowering in short-day 'Carmine' strawberry. HortScience 41: 480-481.

Takeda, F., D. M. Glenn and G. W. Stutte. 2008. Red light affects flowering under long days in a short-day strawberry cultivar. HortScience 43: 2245-2247.

Tang, M., J. Sun, K. Shimizu and K. Kadota. 2015. Evaluation of methods for differential expression analysis on multi-group RNA-seq count data. BMC Bioinformatics 16: 361. DOI: 10.1186/s12859-015-0794-7.

Taoka, K., I. Ohki, H. Tsuji, K. Furuita, K. Hayashi, T. Yanase, M. Yamaguchi, C. Nakashima, Y. A. Purwestri, S. Tamaki, Y. Ogaki, C. Shimada, A. Nakagawa, C. Kojima and K. Shimamoto. 2011. 14-3-3 proteins act as intracellular receptors for rice Hd3a florigen. Nature 476: 332-335.

Teotia, S. and G. Tang. 2015. To bloom or not to bloom: role of microRNAs in plant flowering. Mol. Plant 8: 359-377.

Tian, T., Y. Liu, H. Yan, Q. You, X. Yi, Z. Du, W. Xu and Z. Su. 2017. agriGO v2.0: a GO analysis toolkit for the agricultural community, 2017 update. Nucleic Acids Res. 45: W122W129.

Ueki, M., T. Mochizuki and K. Takano. 1993. Effects of the day and night temperature during short-day and low night temperature treatment of flower bud initiation of strawberry. Bull. Tochigi Agr. Exp. 40: 83-88 (In Japanese with English abstract).

Wang, Y. and D. Deng. 2014. Molecular basis and evolutionary pattern of GA-GID1-DELLA regulatory module. Mol. Genet. Genomics 289: 1-9.

Wang, H. and H. Wang. 2015. Multifaceted roles of FHY3 and FAR1 in light signaling and beyond. Trends Plant Sci. 20: 453-461.

Weigel, D., J. Alvarez, D. R. Smyth, M. F. Yanofsky and E. M. Meyerowitz. 1992. LEAFY controls floral meristem identity in Arabidopsis. Cell 69: 843-859.

Yant, L., J. Mathieu, T. T. Dinh, F. Ott, C. Lanz, H. Wollmann, X. Chen and M. Schmid. 2010. Orchestration of the floral transition and floral development in Arabidopsis by the bifunctional transcription factor APETALA2. Plant Cell 22: 2156 2170 . 